\title{
The Role of Gilts in Transmission Dynamics of Swine Influenza Virus and Impacts of Vaccination Strategies and Quarantine Management
}

\author{
Pia Ryt-Hansen ( $\square$ piarh@sund.ku.dk) \\ Henriette Guldberg Nielsen \\ University of Copenhagen: Kobenhavns Universitet \\ Simon Smed Sørensen \\ University of Copenhagen: Kobenhavns Universitet \\ Inge Larsen \\ University of Copenhagen: Kobenhavns Universitet \\ Charlotte Sonne Kristensen \\ SEGES pig research centre \\ Lars Erik Larsen \\ University of Copenhagen: Kobenhavns Universitet
}

University of Copenhagen https://orcid.org/0000-0003-4819-6869

Research Article

Keywords: Swine influenza A virus, enzootic infections, gilts, quarantine, biosecurity, vaccination, management

Posted Date: October 28th, 2021

DOI: https://doi.org/10.21203/rs.3.rs-1004302/v1

License: (c) (i) This work is licensed under a Creative Commons Attribution 4.0 International License. Read Full License 


\section{Abstract}

Along with an expanding global swine production, the commercial housing and management of swine herds, provide an optimal environment for constant circulation of swine influenza virus (swIAV), thereby challenging farmers and veterinarian in determining optimal control measures. The aim of this study was to investigate the role of gilts in the swIAV transmission dynamics, and to evaluate the impact of different control measures such as quarantine and gilt vaccination.

The study was conducted as a cross-sectional study in ten Danish sow herds, including five swIAV vaccinated and five unvaccinated herds. Blood- and nasal swab samples of gilts, first parity sows, and piglets were collected in different stable units of the production system and analyzed for the presence of SwIAV and swIAV antibodies. Associations between the detection of swIAV, seroprevalence, antibody levels, quarantine measures and vaccination strategy were thereafter investigated to identify possible risk factors for swIAV introductions and persistence within the herds.

Nine of the ten herds had gilts or litters of first parity sows testing positive for SWIAV, and swIAV was detected in both the quarantine, mating- and farrowing unit. The seroprevalence were generally higher in the vaccinated herds, but swIAV was still present in nasal swabs from both gilts and piglets in these herds. Notably, the results revealed that having positive gilts in the end of the quarantine increased the risk of having positive one-week-old litters in the farrowing unit by 2.5 times. These results underline that gilts are important contributors to the continuous circulation of swIAV. Additionally, the recorded vaccination schedules along with quarantine and biosecurity measures were far from optimal emphasizing a needed focus on these factors if control of pathogens such as SwIAV is desired.

\section{Background}

During the last ten years, several studies have described the herd-level persistence of swIAV as a consequence of the expanding swine production favoring large-scale production systems ${ }^{1-7}$. These production systems, if not applying very strict biosecurity measures to avoid mixing of age groups etc., provides a constant weekly flow of naïve individuals ensuring continuous swIAV circulation ${ }^{1-3,8}$. Only few studies have investigated the role of gilts in the within-herd persistence of swIAV7,9-12. However, gilts potentially play a major role for novel swIAV introductions as the majority of the sows-herds only introduce animals from an outside source in relation to replacement of the breeding stock $^{7,13}$. Moreover, if the gilts are not immunized properly before entering the sow herd, they may be naïve to the circulating swIAV herd strain, thereby contributing to the swIAV persistence ${ }^{8}$. Two previous studies have shown correlations between introduction of gilts and an increase risk of herd-level persistence of swIAV ${ }^{9,10}$. To the author's knowledge, however, none of the previous studies sampled gilts at different stages of the production system, and further associated the detection of swIAV at the different stages with the herd vaccination strategy and quarantine measures. Therefore, the present study was designed to include sampling in the quarantine, gestation -, mating and farrowing unit along with collection of detailed information on the gilt vaccination- and quarantine strategies of the herds. This study contributes with important considerations for the veterinarians when deciding optimal control measures for limiting persistence of swIAV in the swine herds and provide new insights into the role of the gilts in the swIAV transmission dynamics.

\section{Results}

SWIAV antibody and sWIAV prevalence in the different stable units

The results of the IAV antibody ELISA and the real-time RT PCR for IAV of the different stable units are summarized in Table 1 and Figure 1.

Among the ten herds included in the study two herds had swIAV positive gilts just after arrival in the quarantine, whereas six herds had swIAV positive gilts at the end of the quarantine. The seroprevalence just after arrival varied greatly between herds, from $95 \%$ of the gilts being seropositive in Herd 5 to $0 \%$ being seropositive in Herd 7. A higher seroprevalence at the end of quarantine period was observed in six of the ten herds, whereas four herds either had a lower level $(n=3)$ or remained at the same level $(n=1)$. In the mating unit, in general, a low prevalence of SwIAV positive gilts were present as four herds had swIAV positive gilts at a $5 \%$ prevalence. The seroprevalence of the pregnant gilts in the gestation unit, were generally high ranging from 55-100\% seropositive gilts and similar levels were observed among the first parity sows in the farrowing unit ranging between 40 to $100 \%$. In the farrowing unit, however, three herds had swIAV positive first parity sows, and in addition, six of the ten herds had swIAV positive one-week old litters in the farrowing unit.

The herds having swIAV positive gilts at the end of the quarantine $(n=6)$ had a significant higher mean prevalence of virus positive oneweek-old piglets in the farrowing unit compared to herds having swIAV negative gilts $(n=4)$ at the end of the quarantine $(15.8 \%$ vs $6.2 \%$, $\mathrm{p}=0.047$ ) and the relative risk of having virus positive one-week-old piglets was $2.5,95 \% \mathrm{Cl}[1.03,6.37]$ for herds that had virus positive gilts 
in the end of the quarantine compared to herds where the gilts tested negative at the end of the quarantine. It should be noted that especially Herd 4 and Herd 10 contributed to this correlation as they had high prevalence of swIAV positive piglets (35\% and $45 \%$ ).

\section{Differences between vaccinated and unvaccinated herds}

Herds 1-5 did not vaccinate against swIAV. Four out of five of these unvaccinated herds had swIAV in at least one of the sampled stable units. In Herd 2 and Herd 5, circulation of swIAV was observed among the one-week-old piglets only, with $10 \%$ and $15 \%$ of the sampled litters testing positive. In Herd 4, $35 \%$ of the one-week-old litters were positive for swIAV, and, interestingly, this herd also had $20 \%$ of the gilts testing positive at the end of the quarantine period. Herd 1, which was a newly started herd with a new breeding stock, had $30 \%$ of the gilts positive for swIAV by the end of the quarantine, posing a high risk for swIAV introduction into the newly established herd. It was therefore decided to take additional samples within this sow-herd approx. three months later to investigate if the virus had spread from the quarantine to the sow-herd (farrowing unit). Interestingly, these "follow-up" samples revealed that 9/12 pools obtained in the farrowing unit was positive for swIAV, including pigs from one- to four-week-of-age.

Herds 6-10 all vaccinated against swIAV, both targeting the gilts and the sows (Table 2). All five vaccinated herds had circulation of swIAV in at least two different stable units and, in general, the herds had virus circulation widely distributed throughout the different stable units (Table 1 and Figure). Four of the five vaccinated herds had swIAV circulating in the quarantine. Herd 6 and Herd 9 had swIAV circulating in both the beginning (at $50 \%$ and $10 \%$ prevalence) and the end of the quarantine (at $15 \%$ and $5 \%$ prevalence), whereas Herd 8 and 10 only had swIAV circulating in the end of the quarantine (at $40 \%$ and $5 \%$ prevalence). Herd 8 , Herd 9 and Herd 10 all had, in addition to swIAV in the quarantine, swIAV circulating in the litters farrowing unit. Additionally, Herd 7, Herd 9 and Herd 10 had swIAV positive sows present in the farrowing unit. Finally, four of the five vaccinated herds had swIAV positive gilts, at a low prevalence (5\%) in the mating unit.

The seroprevalence in the beginning of the quarantine in the unvaccinated herds ranged from $25 \%$ in Herd 4 to $95 \%$ in Herd 5 . By the end of the quarantine the seroprevalence ranged from 20 to $100 \%$. In the gestation unit the prevalence ranged from $55-95 \%$ and was comparable to the seroprevalence observed in the sows of the farrowing unit ranging between 40-100 \%.

In the vaccinated herds the seroprevalence was noticeably different compared to the unvaccinated herds. In Herd 7 none of the sampled gilts of the beginning of the quarantine had any swIAV antibodies, and likewise the seroprevalence was very low in Herd 6 (15\%). Conversely, Herd 8, Herd 9 and Herd 10 had seroprevalences ranging from 55-70 \%. A markedly higher seroprevalence was observed in Herd 7, Herd 8 and Herd 10 by the end of the quarantine, with Herd 8 and Herd 10 reaching $100 \%$ seroprevalence, whereas Herd 7 only reached $85 \%$. Interestingly, both Herd 7 and Herd 10 performed a basic vaccination against swIAV in the quarantine. Low seroprevalences in the end of the quarantine was observed in Herd 6 and Herd 9 reaching only $25 \%$ and $20 \%$, respectively. In the gestation unit, on the other hand, the results were more homogeneous, with all five herds having a seroprevalence ranging between $80-100 \%$. In the farrowing unit similar high seroprevalences was observed ranging between $85-100 \%$, affirming the use of vaccinations in these five sow herds.

As the sample size of vaccinated and unvaccinated herds were rather low, no statistically calculations was performed to evaluate the impact of swIAV vaccination on viral detection and seroprevalence. However, some interesting tendencies were observed. First of all when summing the results of the five unvaccinated and five vaccinated herds, respectively and comparing the overall seroprevalence at the different samplings, it was evident that level of seropositive gilts/sows were lower and fluctuated more in unvaccinated herds compared to the vaccinated herds. On the other hand, the vaccinated herds had a markedly higher seroprevalence in the gilts/sow in the gestation and farrowing unit, compared to the unvaccinated herds, confirming the use of vaccination to stimulate antibody production. However, in regards to circulation of swIAV in the one-week old litters of farrowing unit, the results were similar in vaccinated and unvaccinated herds.

Moreover, none of the gilts/sows of the unvaccinated herds were positive for swIAV in the mating and farrowing compared to four and three of the vaccinated herds having positive gilts/sows in the two units, respectively. Furthermore, only two of the unvaccinated herds had swIAV circulation at the end of the quarantine, compared to four of the vaccinated herds.

\section{Antibody level}

The first parity sows in the farrowing unit having a swIAV positive litter, had significantly lower S/N values compared to the first parity sows having a swIAV negative litter $(0.16$ vs 0.28 , p-value $<0.05)$, indicating that a higher amount of swIAV antibodies present in the first parity sows with a swIAV positive litter.

\section{Vaccinations strategies}

An overview of the different vaccination strategies are summarized in Table 2 . Though all the vaccinated herds had a strategy for gilt vaccination, only $2 / 5$ herds performed double swIAV vaccination of gilts (basic vaccination) before they were introduced into the sow herd. 
In all of the vaccinated herds, additional vaccines were applied at the same time as the influenza vaccine. In one herd (herd 10), the swIAV vaccine was given as the same time as a live attenuated vaccine against PRRSv, whereas the other herd performed vaccinations with up to three different inactivated vaccines at the same time. The mass sow vaccinations against swIAV were in all the herds performed three times per year and also included the gilts.

\section{Level of biosecurity and quarantine management}

All of the herds had personnel inspecting the quarantine in the afternoon, as either minimum 12 hours of quarantine was obliged afterwards or a bath. All herds had separate entrance for entry into the quarantine and in 9/10 of the herds, change of clothes was required before entry. However, change of boots and hand washing were not performed in all herds (Table 3). In five of the ten herds the quarantine stable/s was as an extension to the sow herd or to the weaner unit, and in one herd the "quarantine" was just a pen inside the finisher unit. Five of the herds had a continuous intake of gilts, with one herd introducing gilts on a weekly basis after the quarantine period was finished. Only five of the herds performed washing of the quarantine before introduction of new gilts, and 8/10 herds did have an "empty period" of minimum 3 days before entry of new gilts. For the remaining two herds, one had no empty period and one had an empty period varying between 1-14 days. Vaccination against IAV of personnel was not recommended by the employer and not performed routinely in any of the herds.

\section{Sequencing}

From six (four non-vaccinated and two vaccinated) of the nine swIAV positive herds, HA and NA sequences were obtained to determine the subtype, and compare the sequences to the corresponding vaccine strains included in Respiporc FLU3 ${ }^{14}$ (Table 4 ). In the remaining three herds, the viral load was too low to obtain high quality sequences. The results of the comparison revealed that one of the vaccinated herds were applying a non-matching vaccine, as the subtype was H1pdm09N1av, where at the HA component should be targeted by H1N1pdm09 vaccine strain of Respiporc FLUpan ${ }^{15}$. In the other vaccinated herd, (Herd 10), a $90 \%$ and $88 \%$ sequence identity to the HA and NA proteins included in the Respiporc FLU3 was revealed. Among the three non-vaccinated herds all had subtypes with an Eurasian-avian like HA protein, thereby making the best fitting vaccine choice the Respiporc FLU3. In Herd 1 the gilts tested positive for the H1N1pdm09. Interestingly, the pools obtained three months later from piglets in the farrowing unit, also revealed presence of the $\mathrm{H} 1 \mathrm{~N} 1 \mathrm{pdm} 09$, thereby documenting that the swIAV positive gilts of the quarantine had introduced H1N1pdm09 into the sow herd.

\section{Discussion}

The results of this study clearly emphasize that gilts are an important part of the swIAV transmission dynamics, and that the presence of swIAV and swIAV antibodies in gilts were influenced by the vaccination strategy, quarantine management and biosecurity level.

Circulation of swIAV was documented in nine of the ten investigated sow herds regardless of the use of vaccines to control swIAV. Seven of the herds had gilts/first parity sows testing positive for swIAV, and in six of herds swIAV was circulating in the end of the quarantine period a week before the gilts were introduced into the sow herd. These results underline that gilts should be considered as important for swIAV introductions and the continuous circulation of swIAV in sow herds, and highlights that the management of the quarantine resulted in introduction of swIAV positive animals into the sow herd. Notably, the statistical analysis revealed that having swIAV positive gilt at the end of the quarantine period increased the risk of having swIAV positive one-week-old piglets, thereby emphasizing the risk that poorly managed quarantines can pose to the overall health in the herds and highlights the down-stream consequences. A similar correlation between the introduction of swIAV positive gilts and increased risk of having swIAV positive three-weeks-old piglets was also documented in a previous study $^{10}$. In that study, the relative risk (RR) of having swIAV positive piglets was 1.67 higher in herds introducing swIAV positive gilts compared to those introducing negative gilts, which is slightly lower than the RR of 2.5 documented in our study. The RRs obtained in the studies provide an explanation for why limiting the number of gilt introductions can reduce the enzootic level of swIAV in the herd, as previously suggested by mathematical modelling ${ }^{9}$. The model indicated that extending the time between introductions of replacement gilts to every six month significantly reduced the risk of enzootic swIAV infections. Therefore limiting the number of gilt introductions should be considered key tools in the control of enzootic swIAV as well as avoiding swIAV positive gilts in the end of the quarantine. This risk could be further reduced by applying a basic vaccination of gilts before leaving the quarantine.

Five of the included herds "opened up" their quarantine after a certain number of weeks and then introduce the gilts continuously into the sow herd. Generally, the gilts of the quarantine should be regarded as having the poorest health status in the beginning of the quarantine, and having the highest health status by the end of the quarantine. Therefore there is a significant risk of swIAV introductions after opening the quarantine, and the health status after the first batch of gilts have been introduced into the sow herd is potentially poor. 
In six of the ten herds, the quarantine was an extension to the sow herd or the nursery. In one herd the quarantine was in a stable where slaughter pigs were also housed and continuously introduced from the nursery. The location and the definition of what a quarantine imply is imperative to improve the biosecurity and gilt-management in the herd. The fact that six herds had swIAV circulating in gilts at the end of the quarantine also highlight that the biosecurity and management of the quarantine was not conducted appropriately and that swIAV was introduced into the quarantine from an outside source, probably trough the personnel.

The presence of SwIAV in the beginning of the quarantine could be a result of the gilts originating from a swIAV positive breeding herd, or of the management of the quarantine ensuring transfer of swIAV from the sow herd or infection of the new arrivals of virus persisting in the environment ${ }^{16}$. An increased risk of the latter, should be expected in the herds where no or few "empty days" where implemented before receiving new gilts, and in herds were washing of the quarantine was not performed. The risk of transferring swlAV from the sow herd to the quarantine should be considered in the herds where the location of the quarantine is in connection to the sow herd, nursery or finisher unit, and in the herd with poor biosecurity ${ }^{17,18}$. However, if the quarantine is managed properly and a high level of internal biosecurity in present on the farm, there should be a limited risk of introducing swIAV in the beginning of the quarantine e.g. from the gilt-supplier. In such a situation swIAV will infect all the naive gilts of the quarantine and thereby provide natural immunization, and if the quarantine period is longer than minimum four weeks swIAV should have stopped circulating before the gilts are introduced into the sow herd. However, as seen in two of the included herds of the study, this was not achieved as swIAV was circulating both in the beginning and at the end of the quarantine period. In case a herd utilize the same supplier for purchasing gilts it would be recommend to obtain information on the swIAV status (pos/neg) and which lineage/s are present in addition to information swIAV vaccine protocol. Thereby the risk of introducing a novel subtype into the sow herd could be evaluated and the vaccination policy updated taking into account previous swIAV vaccinations.

Only two of the vaccinated herds had a complete basic vaccination protocol of the gilts while still in the quarantine, whereas the remaining three herds performed the second boost after the gilts were already transferred to the sow herd. Introducing swIAV seronegative gilts or gilts with low antibody titers into the herds, will contribute to the enzootic circulation of swIAV. Conversely, if applying both the prime and boost vaccinations in the quarantine, the gilts will have a high level of swIAV antibodies at the entry into the sow herd, decreasing their risk of contributing to the swIAV transmission. However, if performing two vaccinations in the quarantine one should consider the duration of immunity, which in Europe, for the inactivated vaccines, is ranging between three-six months ${ }^{14,15}$. It will thereby, in many cases, be necessary to apply a third vaccination of the gilts, so they still have high levels of swIAV antibodies before entering the farrowing unit, where swIAV circulation thrive and present a great risk of infection naïve gilts right before farrowing ${ }^{1,8,19}$. This is a critical time for a gilt to become infected as it needs energy for the parturition and the following lactation. Herds having a specific strategy for vaccinating gilts in the quarantine and thereafter including the gilts in the mass sow vaccination program, as many of the herds of this study did, should consider the frequency of the mass sow vaccinations. If mass vaccination is performed one-three times a year, some gilts will not be included in the mass-sow vaccination program before farrowing, and may have increased risk of having low antibody levels before entering the farrowing unit. In that case, it is recommended to have a separate vaccination protocol for the gilts of the sow herd to ensure a third vaccination (after the basic vaccination) thereby avoiding gilts with low levels of swIAV antibodies in the farrowing unit. In line with this, the results of the antibody levels, clearly suggested that the level of antibodies was important in protecting the piglets against swIAV infection as also described several in previous studies ${ }^{20-23}$.

However, in regards to immunization of gilts with inactivated swIAV vaccines, one should consider some potential pitfalls. These include level of homology between vaccine strain and the herd strain, which has been shown to be a potential issue even within swlAVs of the same lineage ${ }^{24-26}$. Moreover, it should be emphasized that the vaccine does not provide sterile immunity ${ }^{13,14}$ and that a certain level of antibodies should be present before a protective effect is observed ${ }^{20-23}$. However, despite the effect on viral shedding may not be complete, the vaccine still can limit the severity of clinical signs, swIAV spread to the lungs and reducing the viral load $24,27-31$

The sequence identities of the herd strains and the "matching" vaccine strains were compared in this study, and the results confirmed the findings of previous studies, that have documented the extensive genetic and antigenic drift that the avian $\mathrm{H} 1 \mathrm{Nx}$ strains has undergone $8,25,32-36$. Interestingly, Herd 10 which had swIAV circulating in both the quarantine, mating unit and farrowing unit, showed very poor genetic similarity to the vaccine strain. In addition, one of the vaccinated herds had an HA gene of H1N1pdm09 origin, while vaccinating with Respiporc FLU3, meaning that an un-matched vaccine was used in this herd. In conclusion, lineage determination and sequencing of the herd strain are important tools in vaccine-selection and in evaluating situations with lack of efficiency of the applied vaccine/s.

Unvaccinated herds without swIAV circulation are at high risk for swIAV introductions from an outside source such as gilts, but also through IAV infected personnel, as exemplified in Herd 1. Herd 1 was a newly started herd, which had purchased a new breeding stock. The sows were negative for swIAV at entry in the quarantine, but positive by the end of the quarantine, indicating that swIAV was introduced from an 
outside source. The sequencing revealed that the swIAV infecting the gilt was H1N1pdm09, which was also the most prevalent subtype in the human seasonal influenza season that year ${ }^{37}$, thereby indicating that the virus could have been introduced by an infected employee. Three month later, the sow herd was still infected by the same strain. This case underline the importance of having strict biosecurity measures in place, and emphasize the need of demanding annual IAV vaccinations of all persons entering the herd.

All the vaccinated herds of this study performed swIAV vaccination of their gilts along with other vaccinations. Herd 10 performed vaccination against PRRSv, using a modified live vaccine (MLV). Interestingly, this herd had the highest prevalence of swIAV in the piglets, despite applying a matched vaccine. To the authors knowledge possible interactions between swIAV and PRRSv vaccines have not been investigated. However, another study has revealed that a MLV PRRSv vaccine applied together with an inactivated mycoplasma vaccine had a negative impact ${ }^{38}$, and possible interactions should therefore be investigated between swIAV-and ML vaccines future studies.

In conclusion, despite the drawbacks of the cross-sectional study design including too low sample size to compare effects of vaccination and possible herd-selection bias, many important considerations of how to introduce gilts, manage the quarantine and implementing biosecurity measures and swIAV vaccines in the herd were stressed. In all herds, several improvements could be made and this probably also applies to many other herds in general. At the end of the day, a better quarantine management, increased biosecurity measures and proper vaccination protocols will not only aid in limiting the circulation of swIAV but also several other viral and bacterial pathogens.

\section{Materials And Methods}

\section{Aim}

The aim of this study was to investigate the role of the gilts in the swIAV transmission dynamics, by assessing the prevalence of swIAV and swIAV antibodies throughout the different stable units of a sow herd, with special focus on the quarantine. A secondary aim was to evaluate associations between swIAV vaccination, quarantine managements and biosecurity measures in the herds on the prevalence of swIAV and swIAV antibodies.

\section{Study design}

Ten herds were included in the study based on the following criteria; the herd should have minimum 800 sows to ensure enough gilts for sampling in each unit, the herd should be free from porcine reproductive and respiratory syndrome virus (PRRSV) or have a PRRSV stable sow unit (no clinical signs), the herd should have minimum one quarantine stable and the herd should purchase gilts from an outside source. In addition, five of the herds should perform swIAV vaccination with Respiporc FLU3 and five sow herds should not have performed any swIAV vaccination for at least one year.

The sampling was performed as a cross sectional study in each herd, with the aim of obtaining all samples the same day. However, in seven of the ten herds it was not possible to sample newly introduced gilts in the quarantine and gilts ready to leave the quarantine on the same day. Therefore, two visits were made in these seven herds. The sampling schedule is shown in Figure 2 . Briefly, in each herd, approx. one week after arrival to the quarantine, blood samples and nasal swabs were collected from 20 gilts and the similar samples were obtained from gilts approx. one week before leaving the quarantine. In addition, one week after arrival to the mating unit, 20 nasal swabs were collected from gilts, whereas only blood samples were obtained from 20 gilts approx. one week before leaving the gestation unit. Finally, one week after farrowing, blood samples and nasal swabs were collected from 20 first parity sows and at the same time, together with nasal swabs from five piglets of the litter of each sampled sow, which were pooled in one tube. If more than 20 gilts were present in a specific batch of one of the stable units (quarantine, mating unit, gestation unit and farrowing unit) the sampling was randomized by counting the number of gilts in the batch and dividing the number with the sample size thereby obtaining the sampling frequency. If the outcome was a decimal number, it was rounded up. If there were less than 20 gilts within a batch, gilts from the previous batch (one week younger) were included until reaching 20 . In herd 1 , additional samples were collected three months after the first visit to investigate if the virus identified during the first sampling had spread from the quarantine to the sow-herd (farrowing unit). The additional samples included 12 pooled samples from the farrowing unit from piglets of one, two, three and four week of age, resulting in three pools of five nasal swabs collected from each age group.

Blood was sampled from vena jugularis and stored in vacutainer serum tubes (Becton-Dickinson, Denmark). Nasal swabs were collected from both nostrils with small or large sterile rayon swabs (Medical Wire, UK) and inserted into both nostrils and turned 360 degrees. The nasal swabs were preserved in a $5 \mathrm{~mL}$ Eppendorf tube containing $2 \mathrm{~mL}$ sterile $0.9 \%$ isotonic $\mathrm{NaCl}$. All samples were stored at $5-8^{\circ} \mathrm{C}$ until arriving at the laboratory within 12-48 hours. At arrival at the laboratory, all blood samples were centrifuged at 3000 RPM for ten minutes to 
obtain the sera, which were subsequently frozen until further analysis at $-20^{\circ} \mathrm{C}$. Similarly, all nasal swabs were vortexed and approx. $600 \mu \mathrm{l}$ of each sample were poured into $1.5 \mathrm{~mL}$ Eppendorf tube and stored at $-80^{\circ} \mathrm{C}$ until further analysis.

Analysis of blood samples

Sera were screened for antibodies against the highly conserved nucleoprotein (NP) of IAV using a commercial blocking enzyme-linked immunosorbent assay (ELISA) (IDEXX Influenza A Ab Test, IDEXX Laboratories, Inc.) following the recommended procedure. Samples with a sample-to-negative $(\mathrm{S} / \mathrm{N})$ value $<0.60$ were considered positive for IAV antibodies and samples $\mathrm{S} / \mathrm{N}>0.60$ were considered negative. The individual $\mathrm{S} / \mathrm{N}$ values were used as a measure of the antibody level for subsequent analysis. As the applied ELISA was a blocking ELISA a low $\mathrm{S} / \mathrm{N}$ value indicated high levels of swIAV antibodies.

Test of nasal swabs for SWIAV virus by real-time RT PCR

The nasal swabs were centrifuged and $200 \mu \mathrm{L}$ were transferred to the sample rack and mixed with $400 \mu \mathrm{lLT}$-buffer (QIAGEN, Copenhagen, Denmark) containing 2-mercaptoethanol (Merck, Darmstadt, Germany). Thereafter, all pathogen nucleic acids were extracted from the nasal swabs using the Cador Pathogen 96 QIAcube HT Kit (QIAGEN) automated on the Qiacube HT (QIAGEN) according to instructions from the supplier.

The resulting extractions were subjected to a previous published real-time RT PCR targeting the matrix gene of IAV to determine if the sample was swIAV positive ${ }^{39}$. The real-time RT PCR was run on the Rotor-Gene Q (QIAGEN) using the following program: $50{ }^{\circ} \mathrm{C}, 30 \mathrm{~min} ; 95$ ${ }^{\circ} \mathrm{C}, 15 \mathrm{~min}$; cycling $45 \mathrm{x}\left(95^{\circ} \mathrm{C}, 10 \mathrm{secs}, 60^{\circ} \mathrm{C} 20 \mathrm{secs}, 64^{\circ} \mathrm{C} 1 \mathrm{sec}, 68^{\circ} \mathrm{C} 1 \mathrm{sec}, 72{ }^{\circ} \mathrm{C} 30 \mathrm{secs}\right)$. A positive and negative control were included in all runs, and a sample was considered positive when having a $\mathrm{Ct}$ value $<36$.

The two samples with the lowest Ct value from each herd were selected for Sanger sequencing of the HA and NA gene as previously described ${ }^{19}$. The sequencing data from LGC Genomics (Berlin, Germany) resulting from the forward and reverse primers were contiged and proof-read manually. The primer binding regions were trimmed off manually to generate consensus sequences of the HA and NA gene. For determining the subtype of the samples the consensus sequences were then checked for the closest sequence match using the function "BLAST against NCBI", and aligned using the MUSCLE algorithm ${ }^{40}$ along with a selection of current Danish HA and NA subtypes, which were then subjected to phylogenetic analysis using the function "create neighbor joining tree". Thereafter, the HA and NA sequences were aligned with the vaccine strains of Respiporc FLU3 (accession no. GQ161124, GQ161100 and GQ161104) ${ }^{14}$, and checked for the level of nucleotide and amino acid identity using the function "create pairwise comparison".

\section{Questionnaire}

At the herd visits the farmer was interviewed, and a questionnaire plus a checklist was filled in based on interview and farm observations. The questionnaire concerned recruitment of gilts, the quarantine, handling of gilts in the quarantine and vaccination strategy, which was left unanswered in the five unvaccinated herds. The questionnaire included 12 closed questions (Yes/No or multiple choice) and 16 semi-open questions (i.e. quantitative variables and description of restrictions after quarantine visit) (supplementary material 1). The checklist included information on internal and external biosecurity measures (i.e. change of clothes and boots in each stable unit) and other vaccinations strategies performed in the herd along with antibiotic usage (supplementary material 2).

\section{Statistics}

Descriptive statistics were calculated using Microsoft Excel version 1911. A Fisher's exact test (Graphpad) was used to determine significant differences in prevalence of one week piglets being positive for swIAV from gilts positive or negative for swIAV at the end of the quarantine. To test if there was a correlation between antibody levels ( $\mathrm{S} / \mathrm{N}$ values) in first parity sows in the farrowing unit and having a swIAV positive litter, the normal distribution of $\mathrm{S} / \mathrm{N}$ values was analyzed to determine the type of statistical test suited for the data. As the $\mathrm{S} / \mathrm{N}$ values were not normally distributed, a Mann-Whitney $\mathrm{U}$ tests were performed on the $\mathrm{S} / \mathrm{N}$ data using GraphPad Prism 8 version 8.3.0538. For all analyses, a p-value below 0.05 was considered statistically significant.

\section{Declarations}

\section{Ethics approval and consent to participate}

No ethical approval was needed for this study as it only included sampling similar to routine diagnostics in the herds. All farmer agreed to participate in the study. 
All authors have read and agreed on the final manuscript.

\section{Availability of data and material}

All data is included in the manuscript and supplementary material.

\section{Competing interest}

One author worked for SEGES Danish Pig Research Centre. SEGES conducts private research, innovation and dissemination of knowledge. SEGES covers all aspects of farming and farm management from crops, climate and environment, nature preservation to livestock farming and organic production. Knowledge is disseminated through various channels such as technical and scientific articles in Danish and international journals. SEGES cooperates with a range of private and public research institutes and knowledge dissemination organizations nationally and internationally. Several of SEGES' projects are financed fully or partially by public funding. The authors declare that the research was conducted in the absence of commercial or financial relationships that could be construed as a potential conflict of interest.

\section{Funding}

The study was funded by SEGES Danish Pig Research Centre.

\section{Authors' contributions}

PRH drafted the manuscript, designed the study and aided in the analyses of the data. HGN and SSS obtained all the samples and performed all the analyses and proofread the manuscript. IL helped design the study and assisted in the statistical analysis and proofread the paper. CSK helped design the study and proof-read the manuscript. LEL helped design the study, coordinated the study and proof-read the manuscript.

\section{Acknowledgement}

The authors would like to thank the participating herds and the herd-veterinarians.

\section{References}

1. Ryt-Hansen, P. et al. Longitudinal field studies reveal early infection and persistence of influenza A virus in piglets despite the presence of maternally derived antibodies. Vet. Res. 50, 36 (2019).

2. Simon-Grifé, M. et al. Swine influenza virus infection dynamics in two pig farms; results of a longitudinal assessment. Vet. Res. $\mathbf{4 3}$, 24 (2012).

3. Rose, N. et al. Dynamics of influenza A virus infections in permanently infected pig farms: evidence of recurrent infections, circulation of several swine influenza viruses and reassortment events. Vet. Res. 44, 72 (2013).

4. Allerson, M. W., Davies, P. R., Gramer, M. R. \& Torremorell, M. Infection Dynamics of Pandemic 2009 H1N1 Influenza Virus in a TwoSite Swine Herd. Transbound. Emerg. Dis. 61, 490-499 (2014).

5. Diaz, A. et al. Complete Genome Sequencing of Influenza A Viruses within Swine Farrow-to-Wean Farms Reveals the Emergence, Persistence, and Subsidence of Diverse Viral Genotypes. J. Virol. 91, e00745-17 (2017).

6. Diaz, A. et al. Multiple Genome Constellations of Similar and Distinct Influenza A Viruses Co-Circulate in Pigs During Epidemic Events. Sci. Rep. 7, 11886 (2017).

7. Diaz, A. et al. Association between Influenza A Virus Infection and Pigs Subpopulations in Endemically Infected Breeding Herds. PLoS One 10, e0129213 (2015).

8. Ryt-Hansen, P. et al. Substantial antigenic drift in the hemagglutinin protein of swine influenza a viruses. Viruses (2020) doi:10.3390/v12020248

9. White, L. A., Torremorell, M. \& Craft, M. E. Influenza A virus in swine breeding herds: Combination of vaccination and biosecurity practices can reduce likelihood of endemic piglet reservoir. Prev. Vet. Med. (2017) doi:10.1016/j.prevetmed.2016.12.013. 
10. Chamba Pardo, F. O. et al. Breed-to-wean farm factors associated with influenza A virus infection in piglets at weaning. Prev. Vet. Med. (2018) doi:10.1016/j.prevetmed.2018.10.008.

11. Serafini Poeta Silva, A. P. et al. Biosecurity practices associated with influenza A virus seroprevalence in sows from southern Brazilian breeding herds. Prev. Vet. Med. (2019) doi:10.1016/j.prevetmed.2019.02.013.

12. Simon-Grifé, M. et al. Seroprevalence and risk factors of swine influenza in Spain. Vet. Microbiol. (2011) doi:10.1016/j.vetmic.2010.10.015.

13. Ryt-Hansen, P. et al. Acute Influenza A virus outbreak in an enzootic infected sow herd: Impact on viral dynamics, genetic and antigenic variability and effect of maternally derived antibodies and vaccination. PLoS One 14, e0224854 (2019).

14. IDT Biologika GmbH. Annex I - summary of product characteristics - RESPIPORC FLU3. 1-6

https://www.ema.europa.eu/en/documents/product-information/respiporc-flu3-epar-product-information_en.pdf.

15. IDT Biologika GmbH. Annex 1 - summary of product characteristics - RESPIPORC FLUpan. 1-6

https://www.ema.europa.eu/en/documents/product-information/respiporc-flupan-h1n1-epar-product-information_en.pdf.

16. Lauterbach, S. E. et al. Detection of influenza A virus from agricultural fair environment: Air and surfaces. Prev. Vet. Med. 153, 24-29 (2018).

17. Torremorell, M., Allerson, M., Corzo, C., Diaz, A. \& Gramer, M. Transmission of Influenza A Virus in Pigs. Transbound. Emerg. Dis. 59, 68-84 (2012).

18. Allerson, M. W., Cardona, C. J. \& Torremorell, M. Indirect Transmission of Influenza A Virus between Pig Populations under Two Different Biosecurity Settings. PLoS One 8, e67293 (2013).

19. Ryt-Hansen, P., Larsen, I., Kristensen, C. S., Krog, J. S. \& Larsen, L. E. Limited impact of influenza A virus vaccination of piglets in an enzootic infected sow herd. Res. Vet. Sci. (2019).

20. Renshaw, H. Influence of antibody-mediated immune suppression on clinical, viral, and immune responses to swine influenza infection. Am. J. Vet. Res. 36, (1975).

21. Deblanc, C. et al. Maternally-derived antibodies do not inhibit swine influenza virus replication in piglets but decrease excreted virus infectivity and impair post-infectious immune responses. Vet. Microbiol. 216, 142-152 (2018).

22. Cador, C. et al. Maternally-derived antibodies do not prevent transmission of swine influenza A virus between pigs. Vet. Res. 47, 86 (2016).

23. Loeffen, W. L. ., Heinen, P. ., Bianchi, A. T. ., Hunneman, W. . \& Verheijden, J. H. . Effect of maternally derived antibodies on the clinical signs and immune response in pigs after primary and secondary infection with an influenza H1N1 virus. Vet. Immunol. Immunopathol. 92, 23-35 (2003).

24. Ryt-Hansen, P. et al. Acute Influenza A virus outbreak in an enzootic infected sow herd: Impact on viral dynamics, genetic and antigenic variability and effect of maternally derived antibodies and vaccination. PLoS One (2019) doi:10.1371/journal.pone.0224854.

25. Henritzi, D. et al. Surveillance of European Domestic Pig Populations Identifies an Emerging Reservoir of Potentially Zoonotic Swine Influenza A Viruses. Cell Host Microbe (2020) doi:10.1016/j.chom.2020.07.006.

26. Vincent, A. L. et al. Influenza A virus vaccines for swine. Vet. Microbiol. 206, 35-44 (2017).

27. Rajao, D. S., Anderson, T. K., Gauger, P. C. \& Vincent, A. L. Pathogenesis and Vaccination of Influenza A Virus in Swine. in Current Topics in Microbiology and Immunology vol. 385 307-326 (2014).

28. Haesebrouck, F. \& Pensaert, M. B. Effect of intratracheal challenge of fattening pigs previously immunised with an inactivated influenza H1N1 vaccine. Vet. Microbiol. 11, 239-249 (1986).

29. Van Reeth, K., Labarque, G., Clercq, S. De \& Pensaert, M. Efficacy of vaccination of pigs with different H1N1 swine influenza viruses using a recent challenge strain and different parameters of protection. Vaccine 19, 4479-4486 (2001). 
30. Lee, J. H. Efficacy of swine influenza A virus vaccines against an H3N2 virus variant. Can. J. Vet. Res. 71, (2007).

31. Bikour, M. H. Evaluation of a protective immunity induced by an inactivated influenza H3N2 vaccine after an intratracheal challenge of pigs. Can. J. Vet. Res. 60, (1996).

32. Zell, R. et al. Displacement of the Gent/1999 human-like swine H1N2 influenza A virus lineage by novel H1N2 reassortants in Germany. Arch. Virol. (2020) doi:10.1007/s00705-019-04457-w.

33. Pia Ryt-Hansen, Jesper Schak Krog, Solvej Østergaard Breum, Charlotte Kristiane Hjulsager, Anders Gorm Pedersen, Ramona Trebbien, L. E. L. Co-circulation of multiple influenza A reassortants in swine harboring genes from seasonal human and swine influenza viruses. Elife 10, (2021).

34. Watson, S. J. et al. Molecular Epidemiology and Evolution of Influenza Viruses Circulating within European Swine between 2009 and 2013. J. Virol. 89, 9920-9931 (2015).

35. Lewis, N. S. et al. The global antigenic diversity of swine influenza A viruses. Elife 5, (2016).

36. Trebbien, R. et al. Genetic and biological characterisation of an avian-like H1N2 swine influenza virus generated by reassortment of circulating avian-like H1N1 and H3N2 subtypes in Denmark. Virol. J. 10, 290 (2013).

37. Institut, S. serum. Danish national influenza surveillance. https://www.ssi.dk/aktuelt/nyhedsbreve/influenza-nyt.

38. Thacker, E. L., Thacker, B. J., Young, T. F. \& Halbur, P. G. Effect of vaccination on the potentiation of porcine reproductive and respiratory syndrome virus (PRRSV)-induced pneumonia by Mycoplasma hyopneumoniae. Vaccine (2000) doi:10.1016/S0264410X(99)00395-3.

39. Nagy, A. et al. Development and evaluation of a one-step real-time RT-PCR assay for universal detection of influenza A viruses from avian and mammal species. Arch. Virol. 155, 665-673 (2010).

40. C. Edgar, R. MUSCLE: multiple sequence alignment with high accuracy and high throughput. (2013) doi:10.1.1.318.6508.

\section{Tables}

Table 1 - Percentage of antibody- and virus positive gilts/sows and piglets at the different sampling times. "Ab" indicates the percentage of gilts/sows testing positive for IAV antibodies in the ELISA tests, whereas "virus" indicates the percentage of gilts/sows and piglets testing positive for swIAV in the real-time RT-PCR. Herds 1-5 did not perform vaccination against swIAV, whereas herd 6-10 performed vaccinations against swIAV. 


\begin{tabular}{|c|c|c|c|c|c|c|c|c|c|}
\hline & Quarantin & & Quarantine & & $\begin{array}{l}\text { Mating } \\
\text { unit }\end{array}$ & $\begin{array}{l}\text { Gestation } \\
\text { unit }\end{array}$ & $\begin{array}{l}\text { Farrowing } \\
\text { sows }\end{array}$ & it - & $\begin{array}{l}\text { Farrowing unit - } \\
\text { piglets }\end{array}$ \\
\hline & $A b(\%)$ & virus (\%) & $A b$ & virus & virus & $A b$ & $A b$ & virus & virus \\
\hline & & & $(\%)$ & $(\%)$ & $(\%)$ & $(\%)$ & (\%) & & $(\%)$ \\
\hline Herd 1 & $12 / 20$ & $0 / 20(0)$ & $7 / 20$ (35) & $6 / 20$ & $0 / 20$ & $12 / 20$ & $11 / 20$ & $0 / 20(0)$ & $0 / 20$ \\
\hline & & & & (30) & (0) & (60) & (55) & & (0) \\
\hline Herd 2 & $7 / 20$ (35) & $0 / 20(0)$ & $7 / 20$ (35) & $0 / 20$ & $0 / 20$ & $14 / 20$ & (8/20) 40 & $0 / 20(0)$ & $2 / 20$ \\
\hline & & & & (0) & (0) & (70) & & & (10) \\
\hline Herd 3 & $14 / 20$ & $0 / 20(0)$ & $5 / 20(25)$ & $0 / 20$ & $0 / 20$ & $11 / 20$ & $10 / 20$ & $0 / 20$ & $0 / 20$ \\
\hline & & & & $(0)$ & (0) & (55) & & (0) & (0) \\
\hline Herd 4 & $5 / 20(25)$ & $0 / 20$ & $17 / 20(85)$ & $4 / 20$ & $0 / 20$ & $13 / 20$ & $9 / 20(45)$ & $0 / 20(0)$ & $7 / 20$ \\
\hline & & $(0)$ & & $(20)$ & (0) & (65) & & & (35) \\
\hline Herd 5 & $19 / 20$ & $0 / 20(0)$ & $20 / 20$ & $0 / 20$ & $0 / 20$ & $19 / 20$ & $20 / 20$ & $0 / 20(0)$ & $3 / 20$ \\
\hline & & & & $(0)$ & (0) & (95) & & & (15) \\
\hline Herd 6 & $3 / 20(15)$ & $10 / 20$ & $5 / 20(25)$ & $3 / 20$ & $1 / 20$ & $20 / 20(100)$ & $20 / 20$ & $0 / 20(0)$ & $0 / 20$ \\
\hline & & & & (15) & (5) & & & & (0) \\
\hline Herd 7 & $0 / 20(0)$ & $0 / 20(0)$ & $17 / 20(85)$ & $0 / 20$ & $1 / 20$ & $16 / 20$ & $17 / 20$ & $1 / 20(5)$ & $0 / 20$ \\
\hline & & & & (0) & (5) & (80) & & & (0) \\
\hline Herd 8 & $\begin{array}{l}14 / 20 \\
(70)\end{array}$ & $0 / 20(0)$ & $\begin{array}{l}20 / 20 \\
(100)\end{array}$ & $8 / 20$ & $1 / 20$ & $20 / 20(100)$ & $\begin{array}{l}20 / 20 \\
(100)\end{array}$ & $0 / 20(0)$ & $1 / 20$ \\
\hline & & & & $(40)$ & (5) & & & & (5) \\
\hline Herd 9 & $13 / 20$ & $2 / 20$ & $4 / 20(20)$ & $1 / 20$ & $0 / 20$ & $20 / 20$ & $20 / 20$ & $1 / 20(5)$ & $2 / 20$ \\
\hline & & (10) & & (5) & (0) & (100) & & & (10) \\
\hline Herd & $11 / 20$ & $0 / 20(0)$ & $20 / 20$ & $1 / 20$ & $1 / 20$ & $17 / 20$ & $18 / 20$ & $2 / 20$ & $9 / 20$ \\
\hline & & & & (5) & (5) & (85) & & & (45) \\
\hline
\end{tabular}

Table 2 - Vaccination strategies in vaccinated herds:

\begin{tabular}{|c|c|c|c|c|c|}
\hline & Herd 6 & Herd 7 & Herd 8 & Herd 9 & Herd 10 \\
\hline SwIAV vaccine & Respiporc FLU 3 & $\begin{array}{l}\text { Respiporc FLU } \\
3\end{array}$ & $\begin{array}{l}\text { Respiporc FLU } 3+ \\
\text { Respiporc FLUpan }\end{array}$ & Respiporc FLU 3 & $\begin{array}{l}\text { Respiporc } \\
\text { FLU } 3\end{array}$ \\
\hline Vaccination strategy for gilts & $\begin{array}{l}24 \text { and } 26 \\
\text { weeks-of-age }\end{array}$ & $\begin{array}{l}21 \text { and } 35 \text { days } \\
\text { after entry }\end{array}$ & $\begin{array}{l}26 \text { and } 28 \text { weeks-of- } \\
\text { age }\end{array}$ & $\begin{array}{l}26 \text { and } 28 \text { weeks- } \\
\text { of-age }\end{array}$ & $\begin{array}{l}2 \text { and } 23 \\
\text { days after } \\
\text { entry }\end{array}$ \\
\hline $\begin{array}{l}\text { Basis-vaccination of gilts before } \\
\text { leaving quarantine }\end{array}$ & No & Yes & No & No & Yes \\
\hline $\begin{array}{l}\text { Mass sow vaccination frequency } \\
\text { per year }\end{array}$ & 3 & 3 & 3 & 3 & 3 \\
\hline $\begin{array}{l}\text { Gilts included in the mass sow } \\
\text { vaccination }\end{array}$ & Yes & Yes & Yes & Yes & Yes \\
\hline \multirow{3}{*}{$\begin{array}{l}\text { Other vaccines given at the same } \\
\text { time }\end{array}$} & Porcilis & Erybac Uno, & \multirow[t]{3}{*}{ Parvoruvax } & Porcilis & \multirow{2}{*}{$\begin{array}{l}\text { Porcilis } \\
\text { PRRS, }\end{array}$} \\
\hline & \multirow[t]{2}{*}{ Ery+Parvo+Lepto } & Porcilis Glässer, & & Ery+Parvo+Lepto, & \\
\hline & & $\begin{array}{l}\text { Porcilis PCV M } \\
\text { Hyo }\end{array}$ & & Porcilis Glässer & $\begin{array}{l}\text { Porcilis } \\
\text { PCV }\end{array}$ \\
\hline
\end{tabular}

Table 3 - Herd information including biosercurity measures and qurantine management 


\begin{tabular}{|c|c|c|c|c|c|c|c|c|c|}
\hline & $\begin{array}{l}\text { No. } \\
\text { of } \\
\text { sows }\end{array}$ & $\begin{array}{l}\text { SPF } \\
\text { status }\end{array}$ & $\begin{array}{l}\text { Production } \\
\text { form }\end{array}$ & $\begin{array}{l}\text { IAV } \\
\text { vaccination } \\
\text { of } \\
\text { personnel }\end{array}$ & $\begin{array}{l}\text { No. of } \\
\text { quarantines/sections }\end{array}$ & $\begin{array}{l}\text { Quarantine } \\
\text { location, } \\
\text { entrance } \\
\text { an } \\
\text { biosecurity } \\
\text { measures }\end{array}$ & $\begin{array}{l}\text { Quarantine } \\
\text { length }\end{array}$ & $\begin{array}{l}\text { Cleaning } \\
\text { of } \\
\text { quarantine } \\
\text { between } \\
\text { batches }\end{array}$ & $\begin{array}{l}\text { Intake of } \\
\text { gilts in the } \\
\text { herd }\end{array}$ \\
\hline $\begin{array}{l}\text { Herd } \\
1\end{array}$ & 1500 & Blue & 7kg pigs & $\begin{array}{l}4 / 7 \\
\text { vaccinated }\end{array}$ & $1 / 1$ & $\begin{array}{l}\text { Connected } \\
\text { to the sow } \\
\text { herd. } \\
\text { Separate } \\
\text { entrance }+ \\
\text { change of } \\
\text { boots and } \\
\text { clothes }\end{array}$ & $\begin{array}{l}\text { 8-10 } \\
\text { weeks }\end{array}$ & $\begin{array}{l}\text { Washed } \\
\text { and dried } \\
\text { for } 3 \text { days }\end{array}$ & Continuous \\
\hline $\begin{array}{l}\text { Herd } \\
2\end{array}$ & 1000 & $\begin{array}{l}\text { Blue + } \\
\text { MYC } \\
+ \\
\text { AP12 }\end{array}$ & $30 \mathrm{~kg}$ pigs & No & $1 / 2$ & $\begin{array}{l}2 \mathrm{~km} \text { from } \\
\text { sow herd. } \\
\text { Separate } \\
\text { entrance }+ \\
\text { change of } \\
\text { boots and } \\
\text { clothes }\end{array}$ & 6 weeks & $\begin{array}{l}\text { No } \\
\text { washing } \\
\text { but empty } \\
\text { for 1-14 } \\
\text { days }\end{array}$ & $\begin{array}{l}4 \\
\text { times/year }\end{array}$ \\
\hline $\begin{array}{l}\text { Herd } \\
3\end{array}$ & 1000 & $\begin{array}{l}\text { Blue + } \\
\text { AP12 }\end{array}$ & $\begin{array}{l}\text { Gilts and } \\
\text { boars }\end{array}$ & No & $1 / 2$ & $\begin{array}{l}\text { Connected } \\
\text { to the sow } \\
\text { herd. } \\
\text { Separate } \\
\text { entrance }+ \\
\text { change of } \\
\text { boots and } \\
\text { clothes }\end{array}$ & $\begin{array}{l}\text { 8-10 } \\
\text { weeks }\end{array}$ & $\begin{array}{l}\text { Washed } \\
\text { and dried } \\
\text { for } 3 \text { days }\end{array}$ & $\begin{array}{l}5 \\
\text { times/year }\end{array}$ \\
\hline $\begin{array}{l}\text { Herd } \\
4\end{array}$ & 930 & Blue & $30 \mathrm{~kg}$ pigs & No & $1 / 1$ & $\begin{array}{l}\text { Part of } \\
\text { fattening } \\
\text { unit. } \\
\text { Separate } \\
\text { entrance }+ \\
\text { change of } \\
\text { boots and } \\
\text { clothes }+ \\
\text { hand wash }\end{array}$ & 6-8 weeks & $\begin{array}{l}\text { No } \\
\text { washing } \\
\text { and no } \\
\text { empty } \\
\text { days }\end{array}$ & Continuous \\
\hline $\begin{array}{l}\text { Herd } \\
5\end{array}$ & 1000 & $\begin{array}{l}\text { Blue + } \\
\text { MYC } \\
+ \\
\text { AP12 }\end{array}$ & $30 \mathrm{~kg}$ pigs & No & $1 / 1$ & $\begin{array}{l}50 \text { meters } \\
\text { from sow } \\
\text { herd. } \\
\text { Separate } \\
\text { entrance }+ \\
\text { change of } \\
\text { boots and } \\
\text { clothes }\end{array}$ & 6-8 weeks & $\begin{array}{l}\text { No } \\
\text { washing } \\
\text { but empty } \\
\text { for 1-14 } \\
\text { days }\end{array}$ & $\begin{array}{l}\text { 6-7 } \\
\text { times/year }\end{array}$ \\
\hline $\begin{array}{l}\text { Herd } \\
6\end{array}$ & 2500 & NA & $30 \mathrm{~kg}$ pigs & No & $1 / 1$ & $\begin{array}{l}10 \mathrm{~km} \text { from } \\
\text { the sow } \\
\text { herd. } \\
\text { Separate } \\
\text { entrance }+ \\
\text { change for } \\
\text { clothes }\end{array}$ & $\begin{array}{l}8-10 \\
\text { weeks }\end{array}$ & $\begin{array}{l}\text { Washed } \\
\text { and dried } \\
\text { for 3-14 } \\
\text { days }\end{array}$ & $\begin{array}{l}5 \\
\text { times/year }\end{array}$ \\
\hline $\begin{array}{l}\text { Herd } \\
7\end{array}$ & 1900 & $\begin{array}{l}\text { Blue + } \\
\text { MYC } \\
+ \\
\text { AP12 }\end{array}$ & Ask & No & $2 / 2$ & $\begin{array}{l}4 \mathrm{~km} \text { from } \\
\text { sow herd } \\
\text { but next to } \\
\text { the } \\
\text { nursery. } \\
\text { Separate } \\
\text { entrance. } \\
\text { Change of } \\
\text { clothes } \\
\text { and boots } \\
+ \text { hand } \\
\text { wash }\end{array}$ & 6 weeks & $\begin{array}{l}\text { No } \\
\text { washing } \\
\text { but empty } \\
\text { for } 7 \text { days }\end{array}$ & Continuous \\
\hline $\begin{array}{l}\text { Herd } \\
8\end{array}$ & 860 & NA & 7kg pigs & No & $1 / 1$ & $\begin{array}{l}400 m \\
\text { from the } \\
\text { sow herd } \\
\text { but next to } \\
\text { the } \\
\text { nursery. }\end{array}$ & 8 weeks & $\begin{array}{l}\text { Washing } \\
\text { of } \\
\text { quarantine } \\
\text { and empty } \\
\text { for } 1-7 \\
\text { days }\end{array}$ & $\begin{array}{l}\text { Continuous } \\
\text { - weekly }\end{array}$ \\
\hline
\end{tabular}




\begin{tabular}{|c|c|c|c|c|c|c|c|c|c|}
\hline & & & & & & $\begin{array}{l}\text { Separate } \\
\text { entrance. } \\
\text { Change of } \\
\text { clothes but } \\
\text { no hand } \\
\text { washing }\end{array}$ & & & \\
\hline $\begin{array}{l}\text { Herd } \\
9\end{array}$ & 1000 & $\begin{array}{l}\text { Blue + } \\
\text { MYC } \\
+ \text { AP2 } \\
+ \\
\text { AP12 }\end{array}$ & $30 \mathrm{~kg}$ pigs & No & $1 / 1$ & $\begin{array}{l}\text { Made as } \\
\text { an } \\
\text { extension } \\
\text { to the sow } \\
\text { herd with } \\
\text { no } \\
\text { separate } \\
\text { entrance + } \\
\text { change of } \\
\text { clothes } \\
\text { and booth } \\
+ \text { use of } \\
\text { gloves }\end{array}$ & 11 weeks & $\begin{array}{l}\text { Washed } \\
\text { and dried } \\
\text { for } 5 \text { days }\end{array}$ & $\begin{array}{l}4 \\
\text { times/year }\end{array}$ \\
\hline $\begin{array}{l}\text { Herd } \\
10\end{array}$ & 1050 & $\begin{array}{l}\text { Blue + } \\
\text { MYC } \\
+ \\
\text { PRRS } \\
\text { type } 1\end{array}$ & $30 \mathrm{~kg}$ pigs & No & $2 / 1$ & $\begin{array}{l}\text { Made as } \\
\text { an } \\
\text { extension } \\
\text { to the sow } \\
\text { herd. } \\
\text { Separate } \\
\text { entrance + } \\
\text { change of } \\
\text { clothes } \\
\text { and boot + } \\
\text { hand wash }\end{array}$ & 5-7 weeks & $\begin{array}{l}\text { Washed } \\
\text { and dried } \\
5-10 \text { days }\end{array}$ & Continuous \\
\hline
\end{tabular}

Table 4 - Identity between herd strains and the corresponding vaccine strain of Respiporc FLU3.

\begin{tabular}{|c|c|c|}
\hline Herds & Subtype & Identity to corresponding vaccine strain included in Respiporc FLU \\
\hline 1 & H1N1pdm09 & - \\
\hline \multirow[t]{2}{*}{2} & H1avN2sw & HA protein identity to HA Haselünne/IDT2617/2003: 92.2-92.4\% \\
\hline & & NA protein identity to NA Bakum/IDT1769/2003(H3N2): 89.5-89.7\% \\
\hline \multirow[t]{2}{*}{4} & H1avN2sw & HA protein identity to HA Haselünne/IDT2617/2003: 92\% \\
\hline & & NA protein identity to NA Bakum/IDT1769/2003(H3N2): 89.5\% \\
\hline \multirow[t]{3}{*}{5} & H1avN2hu & HA protein identity to HA Haselünne/IDT2617/2003: 92\% \\
\hline & & NA protein identity to NA Bakum/IDT1769/2003(H3N2): 84.7\% \\
\hline & & NA protein identity to NA Bakum/IDT1833/2000(H1N2): 82.3\% \\
\hline \multirow[t]{2}{*}{9} & H1pdm09N1av & - \\
\hline & & NA protein identity to NA Haselünne/IDT2617/2003: 90.4\% \\
\hline \multirow[t]{2}{*}{10} & H1avN2sw & HA protein identity to HA Haselünne/IDT2617/2003: 90.25\% \\
\hline & & NA protein identity to NA Bakum/IDT1769/2003(H3N2): 88\% \\
\hline
\end{tabular}

\section{Figures}




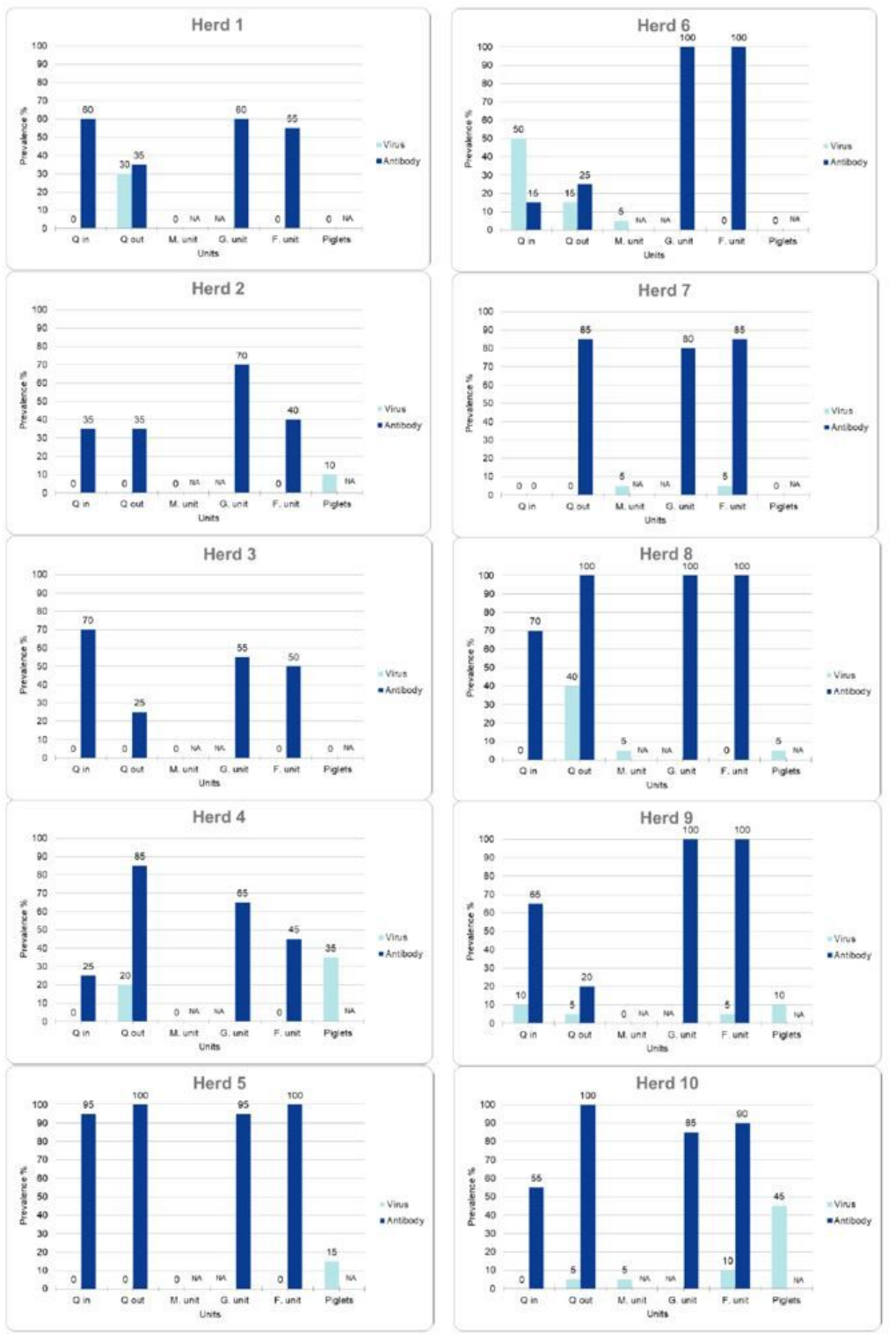

Figure 1

Percentage of antibody- and virus positive gilts/sows and piglets at the different sampling times in each of the ten herds. "Antibody" indicates the presence of antibodies against wwIAV detected by ELISA. "Virus" indicates if the presence of swIAV by real-time RT PCR. "Q in" indicates the sampling time "quarantine in", which was approx. one week after the gilts arrived in the quarantine. "Q out" indicates the sampling time "quarantine out", which was approx. one week before the gilts were introduced into the sow herd. M. unit indicates the sampling time "mating unit", which was approx. one week after the gilts had been introduced into the sow herd. G. unit indicates the sampling time "gestation unit", which was approx. one week before the gilts were transferred to the farrowing unit. F. unit indicates the sampling time of the gilts in the "Farrowing unit", which was approx. one week after farrowing. "Piglets" indicates the same sampling time as the "F. unit", but includes sampling of the one-week-old piglets of the sampled gilts. NA indicates "non-applicable" since no nasal swab or blood sample was obtained at from the sows/piglets in this unit. 


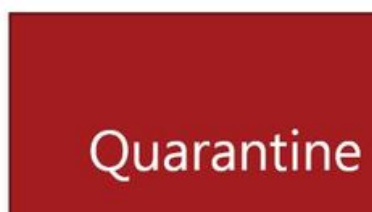

\section{0}

$20 \times$ blood

$20 \times$ nasal swab

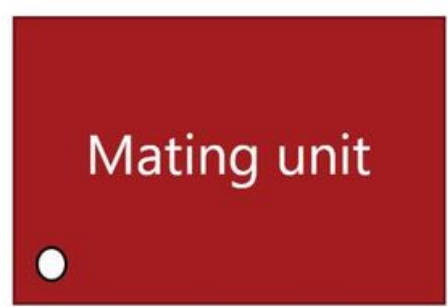

$20 \times$ nasal swab

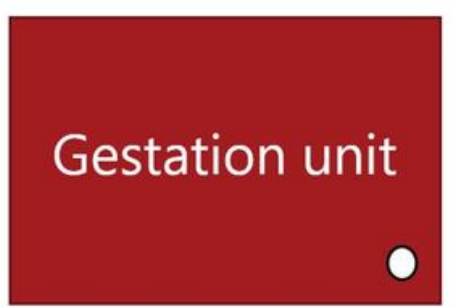

$20 \times$ blood

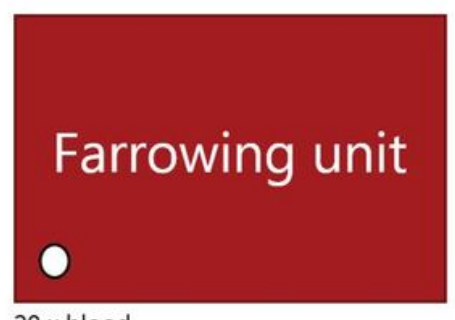

$20 \times$ blood

$20 \times$ nasal swab

$100 \times$ nasal swab piglets (20 pools)

\section{Figure 2}

Overview of sampling in each herd. The figure shows the four different units, which were sampled in each herd, and the type and number of samples obtained from gilts and piglets, respectively. The white dots show when the samples were obtained in each unit being at approx. one week after entry and approx. one week before leaving the quarantine, approx. one week after being transferred to the mating unit, approx. one week before leaving the gestation unit and after approx. one week after farrowing.

\section{Supplementary Files}

This is a list of supplementary files associated with this preprint. Click to download.

- supplementaryfile2.docx

- supplementaryfile1.docx 